\title{
Costly comorbidities of COPD: the ignored side of the coin?
}

\author{
Job F.M. van Boven ${ }^{1,2}$ \\ Affiliations: 'Dept of General Practice, Groningen Research Institute for Asthma and COPD (GRIAC), University \\ Medical Center Groningen, University of Groningen, Groningen, The Netherlands. '2Unit of \\ PharmacoEpidemiology and PharmacoEconomics, Dept of Pharmacy, University of Groningen, Groningen, The \\ Netherlands.
}

Correspondence: Job F.M. van Boven, Dept of General Practice, Groningen Research Institute for Asthma and COPD (GRIAC), University Medical Center Groningen, University of Groningen, Antonius Deusinglaan 1, 9713 AV Groningen, The Netherlands. E-mail: j.f.m.van.bovenđrug.nl

@ERSpublications

Comorbidities more costly than COPD itself: multimorbidity requires change in healthcare practice and financing! http://ow.ly/2fSU30cAGTI

Cite this article as: van Boven JFM. Costly comorbidities of COPD: the ignored side of the coin? Eur Respir J 2017; 50: 1700917 [https://doi.org/10.1183/13993003.00917-2017].

Correct, the alliteration in this editorial's title is not a co-incidence! Ironically, COPD and comorbidity both start with two identical letters and previously in this journal it has even been suggested twice that COPD could also stand for "COmorbidity with Pulmonary Disease" $[1,2]$. However, after having been the only notorious couple for a long time, from now on they will have to face some fierce competition from a new rising kid on the "co"-block: costs.

In the past decade, it has become increasingly clear that chronic obstructive pulmonary disease (COPD) and its comorbidities are almost inseparable, like Blackbeard and his crew of Caribbean pirates, or Hippolyta and her Amazons. Several underlying biological mechanisms and clinical explanations have been suggested to explain this co-existence of multiple diseases besides COPD, including the role of shared risk factors (e.g. tobacco, lifestyle, genetics), chronic systemic inflammation, hypoxia and oxidative stress $[3,4]$. It is therefore no surprise that holistic treatment approaches have been widely advocated with a key role for general practitioners and geriatricians $[3,5]$. Yet, COPD guidelines lagged somewhat behind, with the Global Initiative for Chronic Obstructive Lung Disease (GOLD) providing more explicit guidance on managing some individual comorbidities from 2011 onwards [6]. Even in the latest version [7], it is merely recommended to treat comorbidities as there is no COPD, with no guidance provided on how to organise multimorbidity care. Moreover, most COPD treatment evidence still comes from restricted and controlled COPD trials without significant comorbidity [7]. Real-life data consistently reveal that it is highly relevant to take comorbidities into account from a clinical perspective. Indeed, several previous studies showed their impact on mortality [8,9], exacerbations [10] and hospitalisations [11]. Far less attention has however been paid to the health economic, system and policy implications of the comorbidities of COPD.

In this issue of the European Respiratory Journal, the study of CHEN et al. [12] highlights the substantial financial burden of comorbidity when taking COPD as index disease. In short, Canadian administrative data (1996-2012) from $>250000$ people were used to compare healthcare utilisation and related costs for 16 disease categories in patients with and without COPD. It was found that the excess costs in patients with COPD amounted to $€ 3668$ per person-year of which only one quarter were attributable to COPD, $51 \%$ to comorbidities and $23 \%$ could not be allocated to a specific condition. Most costly comorbidities

Received: May 052017 | Accepted: May 092017

Conflict of interest: Disclosures can be found alongside this article at erj.ersjournals.com

Copyright @ERS 2017 
were circulatory diseases, other respiratory diseases, digestive disorders and psychiatric disorders. Are these new findings? Not really. Generally, these results are largely in line with previous work on this issue [1315] and the comorbidities that are explicitly mentioned in GOLD [7]. This study is however the first that compares the impact of an extensive list of comorbidities to a non-COPD population and uses up to 15 years of follow-up. On the other hand, some noteworthy limitations of the study include the lack of data on COPD severity (e.g. lung function, patient-reported outcomes) and smoke exposure, and that diagnoses were based on claims only. These are all limitations common to administrative data [13], where it remains important to realise that claims data can be prone to under- and overreporting and misdiagnoses [16]. Although being an important part of the economic impact of COPD [17], indirect costs (such as work productivity, or relatives and caretakers care) were not taken into account. Lastly, when interpreting this Canadian study, we should be aware that the transferability of economic costs to other populations relate to differences in local risk factor distributions, healthcare practice, accessibility and other country-specific factors, such as health insurance coverage and reimbursement rules [18].

Despite these limitations, the study of CHEN et al. [12] could be of particular value for health economics and policy. A systematic review of recent cost-effectiveness analyses of new pharmacological COPD treatments already recognised the lack of inclusion of comorbidities in current economic evaluations and recommends inclusion in future analyses [19]. To support these future studies, CHEN et al. [12] provide very useful data. The inclusion of comorbidity costs seems thereby of particular relevance for interventions with expected extra-pulmonary effects such as those that focus on economic evaluations of pulmonary rehabilitation, smoking cessation, indoor and outdoor air pollution prevention or enhancing medication adherence $[20,21]$. Consequently, trials that assess the effectiveness of these interventions need to include not only a broad heterogeneous population, but also a wider range of outcomes.

Looking beyond health economics, on a wider scope, the general messages from COPD comorbidity studies published over the past few years is clear: comorbidities are frequent, have substantial clinical and economic impact and require a holistic treatment approach. When extrapolating the term "comorbidity" to "all multimorbid patients (including those with COPD)" and taking into account the ageing world population, the issue becomes even more relevant. With multimorbidity defined as the presence of at least two long-term morbidities, about one quarter of the total population suffers from multimorbidity, with profound increases with age, but with absolute numbers higher in the under 65 age group [5]. Of note, multimorbidity is usually associated with increased hospitalisation risk, polypharmacy and non-adherence [22]. New National Institute For Health and Clinical Excellence guidelines and recent reviews provide some very useful guidance on the ideal contents of multimorbidity care "beyond the lungs" [23, 24], yet implementation in daily practice will be extremely challenging given the current organisation of care [25]. In my view, but also called for by others [26], some radical changes are first needed in the way that we look at study outcomes and value, practice and organise (respiratory) medicine, and finance our healthcare systems. Theoretically, the required shift from a single disease oriented approach towards a person centred generalist approach is a sine qua non condition. Hereby, we could think of investments in e-health, self-management and monitoring, larger multidisciplinary primary care teams of general practitioners, nurses, pharmacists and physiotherapists, longer primary care consultations, smaller practices, specialists working (or being consulted) in primary care or generalists working in secondary care. In practice, however, health systems are not fully equipped to deal with this shift and there remain numerous obstacles and questions that need to be answered: How do we make sure our staff is sufficiently skilled for managing multimorbidity? How do we identify "at risk" groups that are most likely to benefit? In systems mostly based on fee-for-service, codings and suitable reimbursement for chronic disease management are generally lacking. If bundled payments or population based fees do exist, this may stimulate integrated care, but who will coordinate this care? How do we evaluate economic benefits? All important issues that we are not going to solve as a respiratory community alone, but fundamental issues that should be a top priority for policy makers, healthcare funders and healthcare professionals alike.

Realising that multimorbidity is the norm and not the exception, there is an urgent need for integrated working, organising and financing at all levels of care. From a clinical perspective it has been emphasised over and over again to treat the patient and not the disease. Now, also from a health economic and system point of view, the time has come to flip the coin.

\section{References}

Vanfleteren LE. Does COPD stand for "COmorbidity with Pulmonary Disease"? Eur Respir J 2015; 45: 14-17. Sin DD. Should COPD stand for "comorbidity-related obstructive pulmonary disease"? Eur Respir J 2015; 46: 901-902.

Barnes PJ. Mechanisms of development of multimorbidity in the elderly. Eur Respir J 2015; 45: 790-806.

4 Kent BD, Mitchell PD, McNicholas WT. Hypoxemia in patients with COPD: cause, effects, and disease progression. Int J Chron Obstruct Pulmon Dis 2011; 6: 199-208. 
5 Barnett K, Mercer SW, Norbury M, et al. Epidemiology of multimorbidity and implications for health care, research, and medical education: a cross-sectional study. Lancet 2012; 380: 37-43.

6 Vestbo J, Hurd SS, Agusti AA, et al. Global strategy for the diagnosis, management, and prevention of chronic obstructive pulmonary disease: GOLD executive summary. Am J Respir Crit Care Med 2013; 187: 347-365.

7 Vogelmeier CF, Criner GJ, Martinez FJ, et al. Global Strategy for the Diagnosis, Management, and Prevention of Chronic Obstructive Lung Disease 2017 Report: GOLD Executive Summary. Eur Respir J 2017; 49: 1700214.

8 Divo MJ, Cote C, de Torres JP, et al. Comorbidities and risk of mortality in patients with chronic obstructive pulmonary disease. Am J Respir Crit Care Med 2012; 186: 155-161.

9 Almagro P, Cabrera FJ, Diez-Manglano J, et al. Comorbidome and short-term prognosis in hospitalised COPD patients: the ESMI study. Eur Respir J 2015; 46: 850-853.

10 Westerik JA, Metting EI, van Boven JF, et al. Associations between chronic comorbidity and exacerbation risk in primary care patients with COPD. Respir Res 2017; 18: 31.

11 Van Boven JF, Román-Rodríguez M, Palmer JF, et al. Comorbidome, pattern, and impact of asthma-COPD overlap syndrome in real-life. Chest 2016; 149: 1011-1020.

12 Chen W, FitzGerald JM, Sin DD, et al. Excess economic burden of comorbidities in COPD: a 15-year population-based study. Eur Respir J 2017; 50: 1700393.

13 Mannino DM, Higuchi K, Yu TC, et al. Economic burden of COPD in the presence of comorbidities. Chest 2015; 148: $138-150$

14 Lin PJ, Shaya FT, Scharf SM. Economic implications of comorbid conditions among Medicaid beneficiaries with COPD. Respir Med 2010; 104: 697-704.

15 Huber MB, Wacker ME, Vogelmeier CF, et al. Excess costs of comorbidities in chronic obstructive pulmonary disease: a systematic review. PloS One 2015; 10: e0123292.

16 Triest FJ, Franssen FM, Spruit MA, et al. Poor agreement between chart-based and objectively identified comorbidities of COPD. Eur Respir J 2015; 46: 1492-1495.

17 Van Boven JF, Vegter S, van der Molen T, et al. COPD in the working age population: the economic impact on both patients and government. COPD 2013; 10: 629-639.

18 Vemer P, Rutten-van Mölken MP. Crossing borders: factors affecting differences in cost-effectiveness of smoking cessation interventions between European countries. Value Health 2010; 13: 230-241.

19 Van der Schans S, Goossens LM, Boland MR, et al. Systematic review and quality appraisal of cost-effectiveness analyses of pharmacologic maintenance treatment for chronic obstructive pulmonary disease: methodological considerations and recommendations. Pharmacoeconomics 2017; 35: 43-63.

20 Van Boven JF, Ryan D, Eakin MN, et al. Enhancing respiratory medication adherence: the role of health care professionals and cost-effectiveness considerations. J Allergy Clin Immunol Pract 2016; 4: 835-846.

21 Hoogendoorn M, Feenstra TL, Hoogenveen RT, et al. Long-term effectiveness and cost-effectiveness of smoking cessation interventions in patients with COPD. Thorax 2010; 65: 711-718.

22 Wolff JL, Starfield B, Anderson G. Prevalence, expenditures, and complications of multiple chronic conditions in the elderly. Arch Intern Med 2002; 162: 2269-2276.

23 National Institute for Health and Care Excellence [NG56]. Multimorbidity: Clinical Assessment and Management. www.nice.org.uk/guidance/indevelopment/gid-cgwave0704/documents Date last updated: September 2016.

24 Vanfleteren LE, Spruit MA, Wouters EF, et al. Management of chronic obstructive pulmonary disease beyond the lungs. Lancet Respir Med 2016; 4: 911-924.

25 Royal College of General Practitioners. Managing multi-morbidity in practice... what lessons can be learnt from the care of people with COPD and co-morbidities? Royal College of General Practitioners/National Health Service, 2013.

26 Salisbury C. Multimorbidity: redesigning health care for people who use it. Lancet 2012; 380: 7-9. 